\title{
The WAGASCI experiment at J-PARC to measure neutrino cross-sections on water
}

A. Blondel ${ }^{a}$, F. Cadoux ${ }^{a}$, Y. Favre ${ }^{a}$, B. Martinez ${ }^{a}$, E. Noah ${ }^{* a}$, M. Rayner ${ }^{a}$, M. Antonova ${ }^{b}$, A. Izmaylov ${ }^{b}$, M. Khabibullin ${ }^{b}$, A. Khotyantsev ${ }^{b}$, Y. Kudenko ${ }^{b}$, A. Mefodiev ${ }^{b}, \mathbf{O}$.

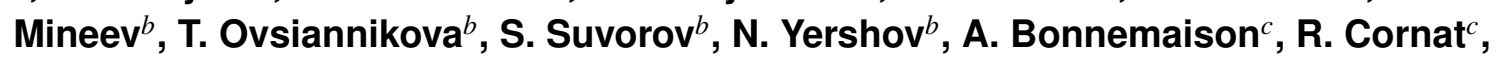

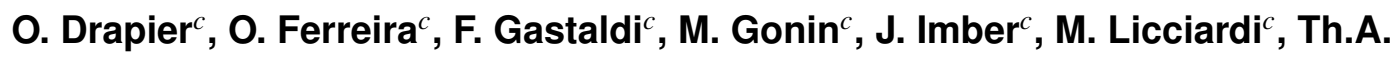
Mueller $^{c}$, T. Ishida $^{d}$, T. Kobayashi ${ }^{d}$, T. Hayashino ${ }^{e}$, A.K. Ichikawa ${ }^{e}$, A. Minamino ${ }^{e}, \mathbf{K}$. Nakamura $^{e}$, T. Nakaya ${ }^{e}$, K. Yoshida ${ }^{e}$, B. Quilian ${ }^{e}$, K. Kin ${ }^{f}$, Y. Seiya ${ }^{f}$, K. Wakamatsu ${ }^{f}$, K. Yamamoto ${ }^{f}$, N. Chikuma $^{g}$, F. Hosomi ${ }^{g}$, T. Koga ${ }^{g}$, M. Yokoyama ${ }^{g}$, Y. Hayato ${ }^{h}$

${ }^{a}$ University of Geneva, Section de Physique, DPNC, Geneva, Switzerland

${ }^{b}$ Institute for Nuclear Research of the Russian Academy of Sciences, Moscow, Russia

${ }^{c}$ Ecole Polytechnique, IN2P3-CNRS, Laboratoire Leprince-Ringuet, Palaiseau, France

${ }^{d}$ KEK, Japan

${ }^{e}$ Kyoto University, Department of Physics, Kyoto, Japan

${ }^{f}$ Osaka City University, Osaka, Japan

${ }^{g}$ The University of Tokyo, Department of Physics, Tokyo, Japan

${ }^{h}$ The University of Tokyo, Institute for Cosmic Ray Research, Tokyo, Japan

E-mail: etam.noahecern.ch

In the T2K experiment, the far detector, Super-Kamiokande, observes neutrino interactions on water while the near detectors are mainly constituted of plastic scintillator. The uncertainty due to the difference of target materials is one of major systematic uncertainties in the T2K neutrino oscillation analyses. A new neutrino detector named WAGASCI has been developed to measure the cross section ratio of neutrino (and antineutrino) interactions with water and plastic targets with a large angular acceptance. The experiment will be situated at the J-PARC near detector station. The water sections of the WAGASCI detector consist of $80 \%$ water within a mesh of 3-mm thick plastic scintillators assembled into a 3D grid-like structure. The scintillator is readout with Wavelength shifting fibers connected to new Multi-Pixel Photon Counters (MPPCs) with low crosstalk rate and high photon detection efficiency (PDE). The experiment is complemented with an instrumented muon range detector comprising a magnetic spectrometer (Baby MIND).

The European Physical Society Conference on High Energy Physics

22-29 July 2015

Vienna, Austria

${ }^{*}$ Speaker. 


\section{Introduction}

Particle accelerator beam-driven neutrino oscillation studies are actively pursued at the T2K experiment [1]. Neutrino signal events registered at the far detector, Super-Kamiokande, located $295 \mathrm{~km}$ from the neutrino source are compared with flux predictions and measurements at a suite of near detectors located $280 \mathrm{~m}$ from the source. The difference in detector target nuclei between the near detector, mainly plastic scintillator, and the far detector which is a large water cherenkov detector, is one of the major systematic uncertainties in neutrino oscillation analyses since the neutrino interaction cross-sections on water are poorly known. A new neutrino detector, the WAter Grid And SCIntillator detector (WAGASCI), is under development aimed at measuring the ratio of neutrino cross-sections on plastic and water to $3 \%$ accuracy, using techniques tried and tested with the INGRID detector [2].

As will be highlighted, recent performance enhancements of silicon photomultipliers (or multipixel photon counters - MPPC), along with fine segmentation of plastic scintillators using thin slabs that provide sufficient light yield, enable a grid design with cells that maximize the water fraction in the main neutrino target whilst providing good acceptance for neutrino events with 3D tracking of outgoing leptons.

\section{WAGASCI detector design}

The WAGASCI experiment will be installed on the B2 floor of the ND280 building at J-PARC, at an angle of 1.6 degrees to the neutrino beam axis, compared to the 2.5 -degree T2K off-axis beam. The energy spectra are thus very similar, with a small shift towards higher peak energies of $0.7 \mathrm{GeV}$ for the WAGASCI beam due to its lower off-axis angle. The WAGASCI detector consists of two main systems:

- a central neutrino interaction target;

- surrounding muon range detectors (MRD).

The above are further sub-divided: the central neutrino interaction target consists of 4 blocks, each $1 \times 1 \times 0.5 \mathrm{~m}^{3}$, with an alternating pattern along the beam direction between water-filled and hydrocarbon-filled blocks motivated by a desire to reduce the flux and MRD acceptance differences in each type of block, whilst the muon range detectors consist of two side MRDs and a magnetized downstream MRD, Figure 1.

The water and hydrocarbon blocks are instrumented with plastic scintillators, a mix of 3D gridlike structures and conventional $\mathrm{x}-\mathrm{y}$ planes of long thin bars. The 3D grid is constructed by locking rows and columns of scintillator bars amongst themselves through grooves machined in a comblike arrangement for that purpose, Figure 2. The resulting cells between the plastic scintillator walls are $5 \times 5 \times 2.5 \mathrm{~cm}^{3}$. These cells are then filled with either water or hydrocarbon. 

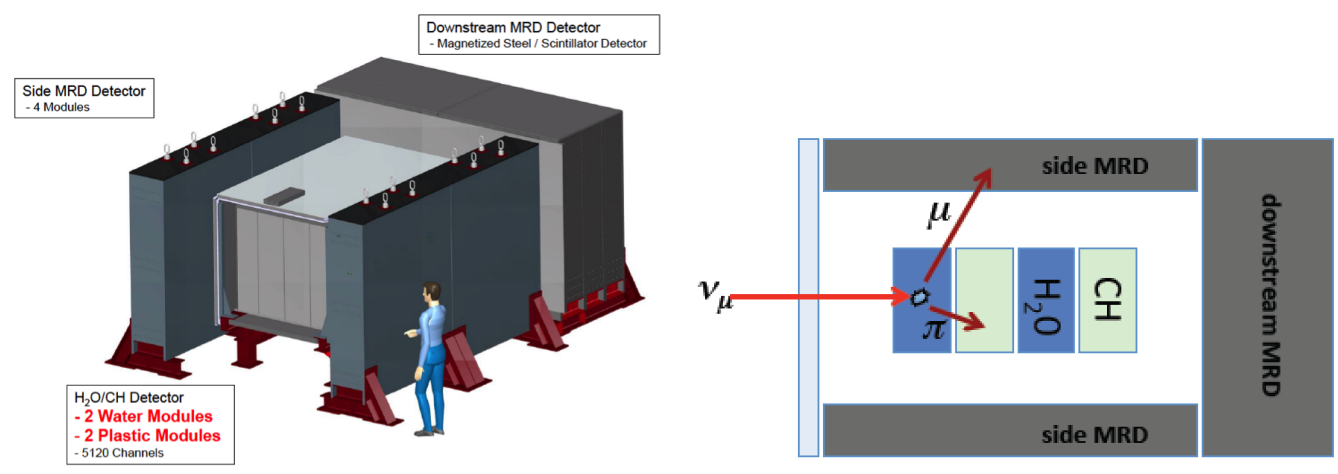

Figure 1: Sketch of the WAGASCI detector. The central $\mathrm{H}_{2} \mathrm{O}(\times 2)$ and $\mathrm{CH}(\times 2)$ neutrino targets are flanked by 2 side muon range detectors (sMRD) and one downstream muon range detector (dMRD - Baby MIND).
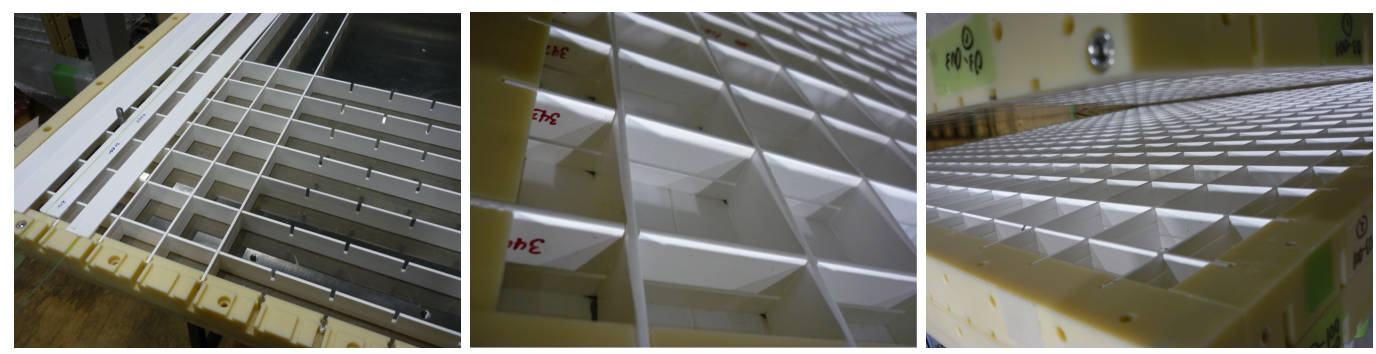

Figure 2: WAGASCI plastic scintillator 3D grid structure. Dimensions of each cell are $5 \times 5 \times 2.5 \mathrm{~cm}^{3}$. The cells are filled with either $\mathrm{H}_{2} \mathrm{O}$ or $\mathrm{CH}$ to form the neutrino interaction target of the experiment.

\section{Central neutrino target MPPCs}

The T2K experiment was a pioneer in the use of MPPC photosensors on a large scale, with 56000 MPPCs of type S10362-13-050C operational since 2009 [3]\&[4]. These were derived from commercial devices, with the sensitive area increased from $1 \times 1 \mathrm{~mm}^{2}$ to $1.3 \times 1.3 \mathrm{~mm}^{2}$ to provide better acceptance for the light emitted from the $1.0 \mathrm{~mm}$ diameter wavelength shifting fiber that collects the light in the plastic scintillator and transmits it to the bar ends where the photosensors are located. Since then, there has been recent marked progress in the performance of such devices, first with the launch of devices with suppression of afterpulse and dark noise in 2013 and then the commercialization of devices with low crosstalk end 2014 (type S13081). These enhanced properties enable operation:

- at higher over voltage due to the lower dark noise rate;

- with much lower signal thresholds due to the lower crosstalk.

Extensive measurements confirm the lower dark noise rate (factor $\times 4$ ) and lower crosstalk $($ factor $\times 5$ ) of the new devices despite the higher over voltage applied (4 V vs $1.1 \mathrm{~V}$ ) [5]. The increase in photon detection efficiency (PDE) is a factor 1.9 and is associated with an increase in gain of factor 3.6.

The light yield for the combination of 3D grid bars and new MPPC was measured using a 600 $\mathrm{MeV}$ positron beam. The experimental setup allowed detailed scans of the light yield as a function 
of position along the grid, showing signals of more than 10 photo-electrons in the least sensitive areas of the scintillator, and detection efficiencies of greater than $99 \%$ for the whole scintillator with a signal threshold set at 1.5 photo-electrons, Figure 3.

Plastic scintillators for the central neutrino detector will be read out with arrays of 32 channel MPPCs in order to have a more compact layout for the 5276 channels.

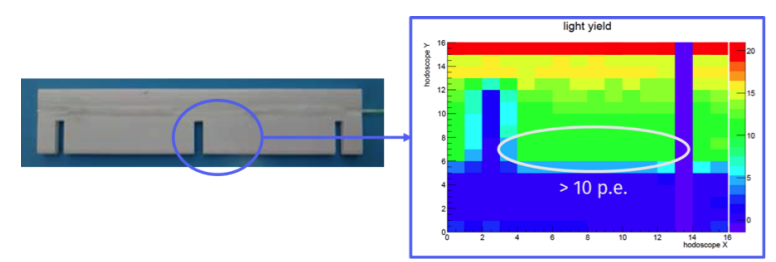

Figure 3: Light yield map measured in photo-electrons for a $3 \mathrm{~mm}$ thick plastic scintillator bar similar to those used in the 3D WAGASCI grid, readout with wavelength shifting fiber and type S13081 MPPC.

\section{Muon spectrometers}

The muon spectrometers are designed to measure the momentum of muons produced from interactions of neutrinos in the central target by range (side MRDs) and both by range and curvature (downstream MRD - Baby MIND). Both types of spectrometers consist of several plates of steel 3 $\mathrm{cm}$ thick, interleaved with plastic scintillator detector modules.

To address charge identification limitations of typical magnetized muon spectrometers for the short range tracks of muons with momenta below $500 \mathrm{MeV}$ which are due to multiple scattering in the steel, the Baby MIND design comprises the following features:

1. the magnetic field is increased to $1.5 \mathrm{~T}$ thanks to the use of high permeability iron combined with a novel magnetization scheme;

2. the measurement of curvature in the first planes of iron is augmented by measurements of the muon track angles in-between the iron plates.

The Baby MIND magnetization concept is based on the principle of flux return used in current transformers to minimize power consumption, to values of order $100 \mathrm{~W}$ per steel module. The flux must return around the left/right edges of the $3.5 \mathrm{~m}$ wide, $2 \mathrm{~m}$ high steel plates, leading to zones where the field is less well defined. The middle part of the steel module where the field is very uniform, and the horizontal component of the field strength accounts for $>95 \%$ of the total field strength, has been extended by design from $43 \%$ for a single slit design to $80 \%$ with a double slit design, Figure 4. It is this area which is instrumented with detector modules.

Detector modules consist of $x-y$ planes of plastic scintillator bars, with wavelength shifting fibers read out with single MPPCs of the ceramic package type. The Baby MIND modules are optimized for the field map, with finer segmentation along the vertical axis $(3 \mathrm{~cm} \times 288 \mathrm{~cm}$ horizontal bars) and coarser segmentation along the horizontal axis (21 cm x $195 \mathrm{~cm}$ vertical bars). The layout of scintillator and steel modules along the beam axis is shown in Figure 5. The scintillator modules are read out with a newly developed front end board [6]. The FEB schematic is shown in Figure 


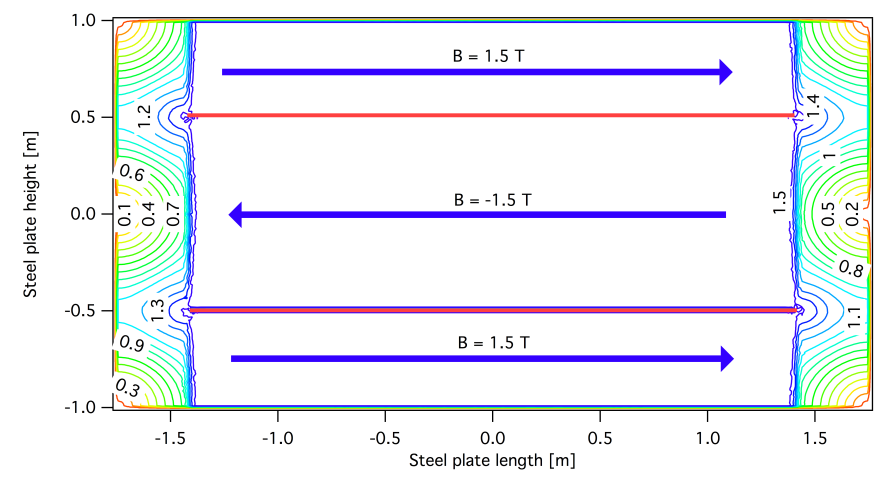

Figure 4: Baby MIND double-slit magnetic field map with a $280 \mathrm{~cm}$ long aluminium coil, $1.5 \mathrm{kA}$.

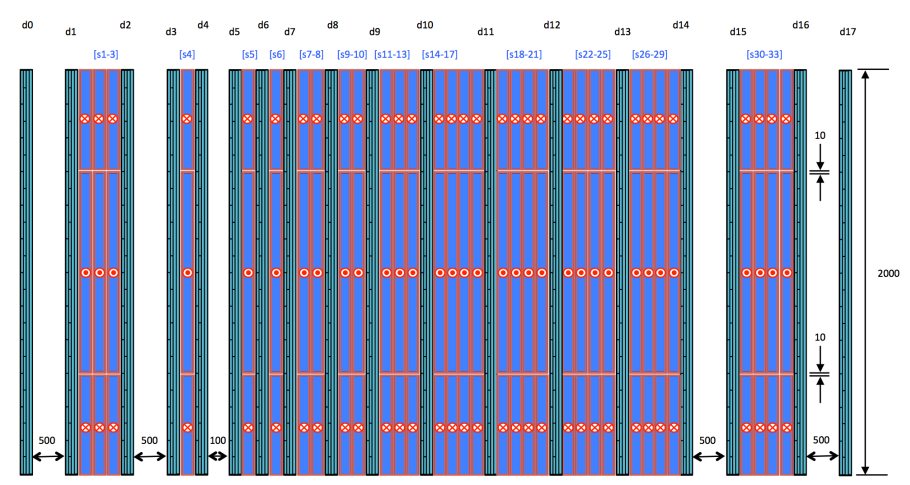

Figure 5: Baby MIND layout showing the positioning of steel (s) and scintillator detector (d) modules along the beam axis for a beam incident from the left.
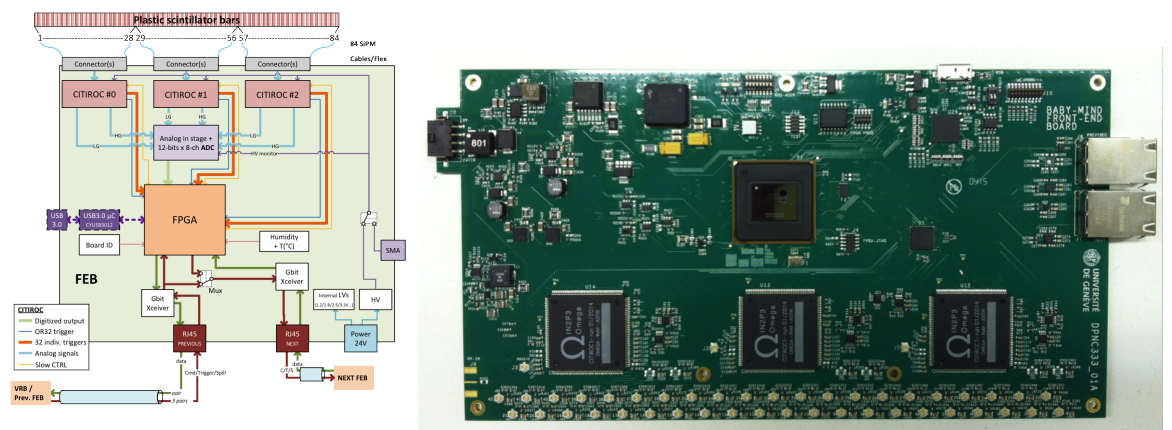

Figure 6: Baby MIND Front End Board (FEB). left) schematic. right) first prototype.

6. It consists of readout ASICs (CITIROC), an ADC for the digitization of the analogue outputs of the CITIROC, an FPGA for time-stamping of trigger outputs and digital processing.

Simulations of the Baby MIND downstream of the WAGASCI central neutrino detector were carried out to study the reduction of wrong sign muon contamination inherent with anti-neutrino beams. In anti-neutrino beam mode, muon neutrinos form a significant fraction of the total neutrino beam, Figure 7. The 90\% charge reconstruction efficiency of the Baby MIND contributes to decreasing the neutrino background down to $2.9 \%$ from $30 \%$ for all neutrino interactions resulting in a muon reaching it. Figure 7 shows an event display for a neutrino interaction in the central neu- 
trino detector and the outgoing muon measured by the Baby MIND spectrometer. A programme of measurements is planned at CERN to characterize the Baby MIND in a charged particle beam prior to its operation at WAGASCI.
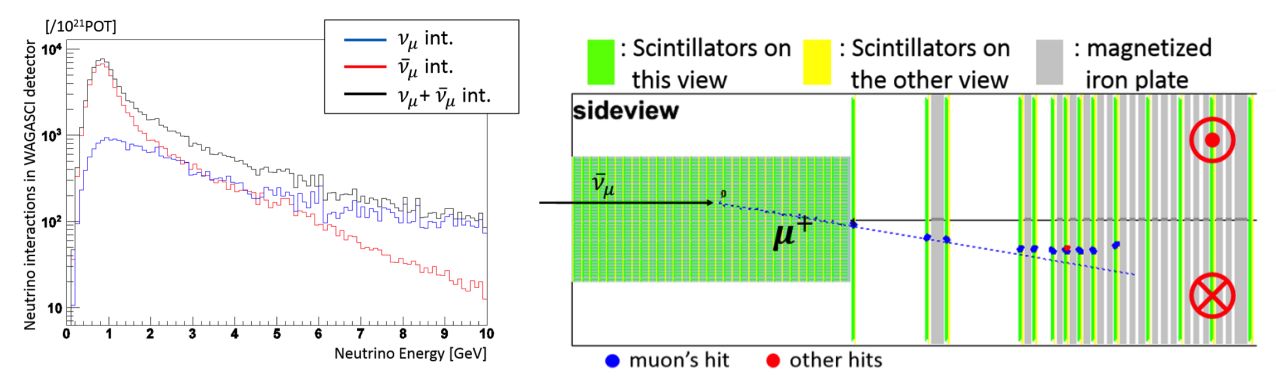

Figure 7: Left) In anti-neutrino beam mode, $v_{\mu}$ interactions in the WAGASCI detector form a significant fraction of the total number of neutrino interactions. Right) Event display showing a neutrino interaction in the WAGASCI central neutrino detector with the outgoing muon trajectory in the Baby MIND downstream.

\section{Summary}

The WAGASCI experiment under construction for operation at the J-PARC neutrino beamline will contribute to lowering systematic uncertainties in T2K neutrino oscillation studies. It consists of a central core of water- and hydrocarbon neutrino interaction targets, flanked on two sides with muon range detectors and with a downstream magnetized muon detector. A novel 3D grid structure of thin plastic scintillators results in cells that can be filled with either water or hydrocarbon. The large $\mathrm{H}_{2} \mathrm{O} / \mathrm{CH}$ volume fraction of up to $80 \%$ of the total volume and event acceptance will lead to measurements of the ratio of water to carbon cross-sections to $3 \%$ accuracy. The downstream Baby MIND magnetized muon spectrometer is designed to improve event selection efficiencies by providing charge identification of muons, especially in anti-neutrino beam mode where the neutrino contamination is as high as $10 \%$ at $0.7 \mathrm{GeV}$ and worsens above this peak neutrino energy.

\section{References}

[1] K. Abe et al. [T2K Collaboration], "The T2K Experiment," Nucl. Instrum. Methods Phys. Res., Sect. A 659, 106 (2011). [arXiv:1106.1238 [hep-ex]].

[2] K. Abe et al. [T2K Collaboration], "Measurement of the inclusive $v_{\mu}$ charged current cross section on iron and hydrocarbon in the T2K on-axis neutrino beam," Phys. Rev. D 90, no. 5, 052010 (2014) [arXiv:1407.4256 [hep-ex]].

[3] A. Vacheret et al.,"Characterization and Simulation of the Response of Multi Pixel Photon Counters to Low Light Levels,” Nucl. Instrum. Meth. A 656 (2013) 69 [arXiv:1101.1996 [physics.ins-det]]

[4] M. Yokoyama et al., "Application of Hamamatsu MPPC to T2K Neutrino Detectors," Nucl. Instrum. Meth. A 610 (2009) 128 [arXiv:0807.3145 [physics.ins-det]].

[5] F. Hosomi et al., "Performance test of new MPPC for a new neutrino detector WAGASCI," PoS PhotoDet 2015 (2015).

[6] E. Noah et al., "Readout scheme for the Baby MIND detector," PoS PhotoDet 2015 (2015). 